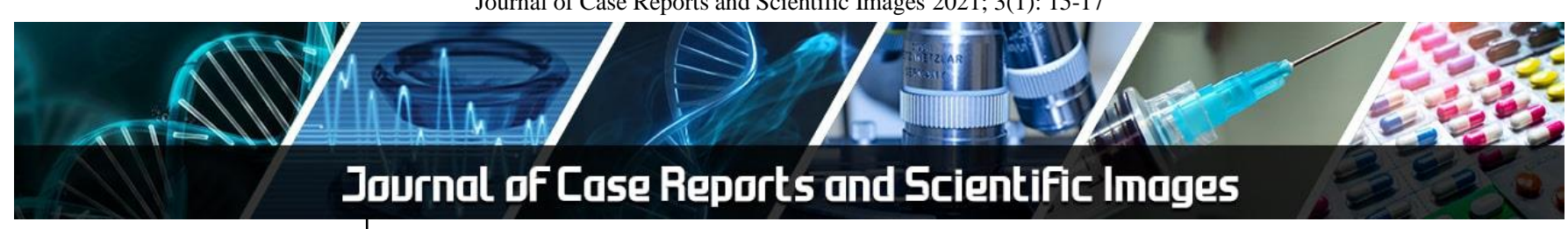

E-ISSN: 2708-0064

P-ISSN: 2708-0056

IJCRS 2021; 3(1): 15-17

www.allcasereports.com

Received: 14-11-2020

Accepted: 19-12-2020

Amit Sharma

Resident, Department of Pathology, INHS Asvini, Colaba, Mumbai, Maharashtra, India

\section{Ritu Mehta}

Consultant Oncopathologist, Department of Pathology,

INHS Asvini, Colaba,

Mumbai, Maharashtra, India

Rakesh Holla

Resident, Department of Pathology, INHS Asvini, Colaba, Mumbai, Maharashtra, India

Naveen Chawla

Head of Department,

Department of Pathology,

INHS, Asvini Colaba,

Mumbai, Maharashtra, India

\section{Gurpreet Kaur}

Consultant Pathologist,

Department of Pathology,

INHS, Asvini Colaba,

Mumbai, Maharashtra, India

Corresponding Author: Amit Sharma

Resident, Department of Pathology, INHS Asvini, Colaba, Mumbai, Maharashtra, India

\section{Actinomycosis lymphadenitis: A rare case report}

\author{
Amit Sharma, Rakesh Holla, Ritu Mehta, Naveen Chawla and Gurpreet \\ Kaur
}

DOI: $\underline{\text { https://doi.org/10.22271/27080056.2021.v3.i1a.26 }}$

\begin{abstract}
Actinomycosis-associated lymphadenopathy is a rare condition that may clinically simulate malignancy hence early and appropriate diagnosis is necessary for patient management.
\end{abstract}

Keywords: Actinomycosis, lymphadenopathy

\section{Introduction}

Actinomycosis is a subacute-to-chronic bacterial infection. It is characterized by contiguous spread, suppurative, granulomatous inflammation and formation of multiple abscesses along with sinus tracts that may discharge characteristic sulfur granules ${ }^{[1]}$. In suspicious lesions FNA cytology may be used for diagnosis in refractory lesions. Microbiologic examination also plays a crucial role in the definitive diagnosis. In histopathological examination of lymph nodes, the presence of capsular thickening with reactive germinal centers, intranodal fibrosis, multiple micro abscesses and scattered neutrophil laden macrophages suggests actinomycosis and multiple sections may be needed for identification of specific sulfur granules. Early and appropriate diagnosis is important for early management of patient.

\section{Case history}

A 53 year old male presented with complaints of swelling in right side of neck since three months. Swelling was painless, not associated with fever, trauma, weight loss and was gradually increasing in size. He was a chronic smoker and a known case of diabetes mellitus, hypertension and was on regular medications.

On local examination a well-defined, non-tender, hard, fixed soft tissue swelling measuring 5 x $6 \mathrm{~cm}$ was palpable at right submandibular region. USG neck was suggestive of necrotic lymph node in right cervical region, level IIa. Patient underwent CECT neck which revealed submandibular lymphadenopathy with enhancing hypodense lesion suggestive of secondary's from unknown primary. ENT evaluation was essentially normal and no suspicious lesion detected in oral and nasal cavity. Oral hygiene of patient was poor with multiple carries and missing teeth.

FNAC from the swelling revealed evidence of haemophagocytosis, thus the possibility of viral/bacterial/fungal infection and malignancy may be considered was opined. After the diagnostic workup, surgical excision of mass was considered. Sialadenectomy of submandibular gland (right) and lymph node excision was done under general anesthesia.

The excision biopsy was sent for histopathological examination. On gross examination we received a lymph node measuring $1.5 \mathrm{x} 1 \mathrm{~cm}$. External surface and cut surface was unremarkable. Haematoxylin and Eosin stained sections from lymph node revealed varied sized lymphoid follicles with dense inflammatory infiltrate comprising of lymphocytes, neutrophils, plasma cells, histiocytes and eosinophils. Few foci show neutrophilic abcesses surrounded by areas of fibrosis. A club shaped cluster of filamentous organism was seen with deposition of radiating eosinophilic material around it as shown in figure 1(a).

Sections from right submandibular salivary gland revealed moderate lymphomononuclear infiltrate surrounding benign salivary ducts and glands suggestive of chronic sialdenitis.

Special stains were performed. The organism stained positive with gram, grocott methenamine silver (GMS) stain, PAS stain and was negative with $\mathrm{ZN}$ satin as shown in figure $1(b, c, d)$. Based on this diagnosis of actinomycosis was made.

\section{Discussion}

Actinomyces is a gram positive, non-motile filamentous branching, anaerobic bacteria ${ }^{[1]}$. It occur as a commensal in the oral cavity and gastrointestinal tract of healthy persons ${ }^{[1]}$. 


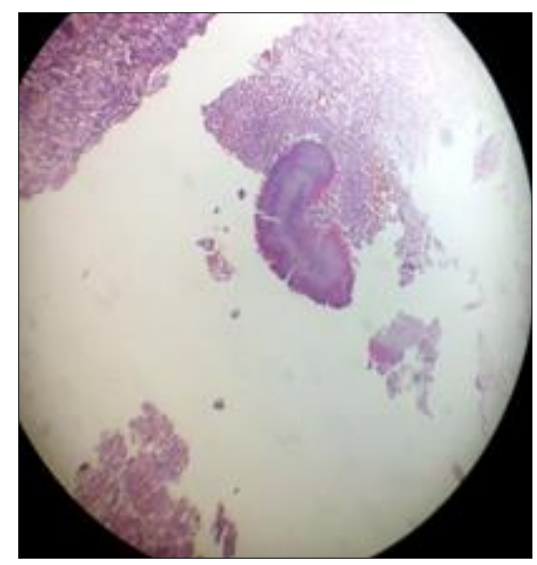

Fig 1a: H \& E stain (10X)

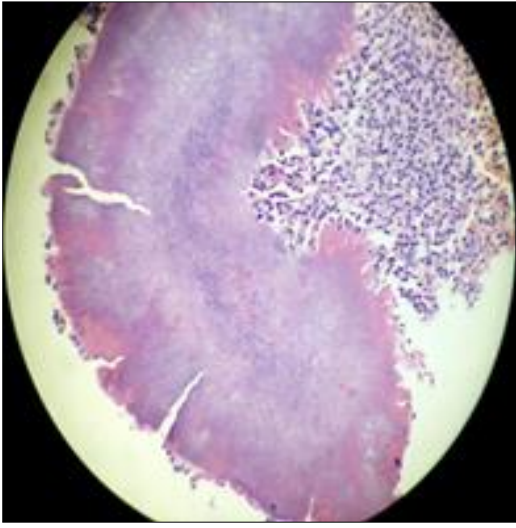

Fig 1b: H \& E stain (20X)

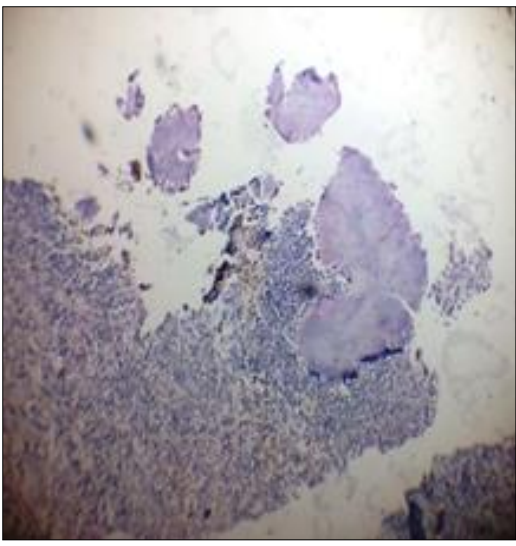

Fig 1c: PAS stain (10X)

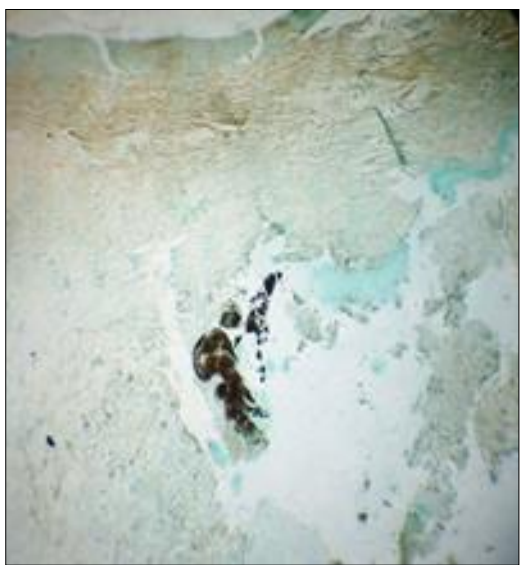

Fig 1d: GMS stain (10X)

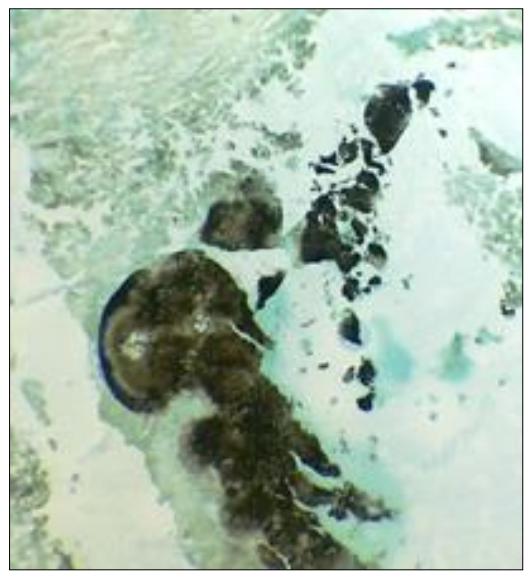

Fig 1e: GMS stain (20X)

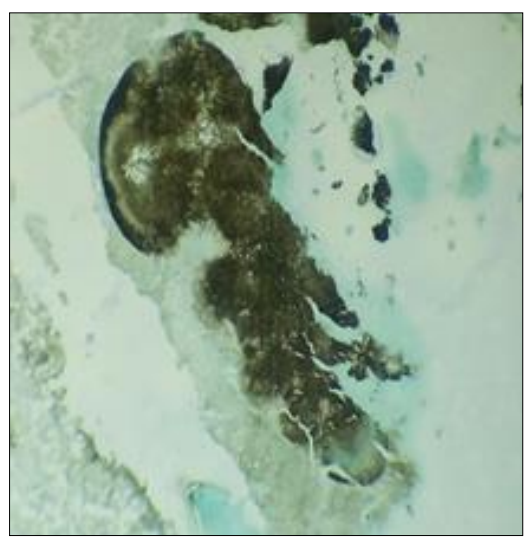

Fig 1f: GMS stain (20X)

It rarely causes disease and very few cases of actinomcycosis lymphadenitis has been reported as depicted in table 1 of our study. The pathogeneic forms do not exist freely in nature therefore humans are themselves the natural reservoirs ${ }^{[5]}$. No person to person transmission occurs ${ }^{[5]}$. Actinomycosis is an indolent, progressive and invasive bacterial infection1.The pathogenesis of actinomycosis is related to the interruption of the mucocutaneous barriers anywhere from mouth to rectum, which leads to suppuration and abscess formation. The abscess then expands into adjacent tissue with no regard to tissue planes. The most common route of spread is local invasion ${ }^{[2]}$. Though hematogenous dissemination can occur, particularly to the brain and lungs, however lymphatic spread is uncommon ${ }^{[1]}$. Our study is consistent with ramyond et al. who says actinomycosis usually found in immunocompetent people as is our case, however it can also occur in patients who are immunocompromised ${ }^{[5]}$.

Four clinical forms of actinomyces exists i.e. cervicofacial, abdominopelvic, thoracic, cerebral ${ }^{[5]}$. Cervicofacial form is most common with frequency of this site being 11-97\%. Cervicofacial actinomycosis is caused by the infection of actinomyces israelii and usually occurs in the oral cavity. Our study is in concordance with moniruddin et al. which says surgery and poor dental hygiene is the most common cause and our case also typically had poor dental hygiene ${ }^{[7]}$. Our case also presented with most common location of cervicofacial region as shown in study by Raymond et al.

Lymphadenopathy is a common clinical finding in many neoplastic and infectious diseases ${ }^{[2]}$. Lymphadenopathy was present in our case however the typical clinical signs and symptoms of cervicofacial actinomycosis such as woody 
fibrosis (characteristic induration), multiple sinus tracts, trismus and presence of sulfur granules were absent. A malignant tumor was initially suspected because of the absence of evident signs of infection and the presence of a rapidly growing tumor-like mass. The clinical findings of this rare bacterial disease are similar to those of certain malignant tumor's. Thus, sometimes it may be difficult to diagnose actinomycosis clinically. In such cases histopathological diagnosis becomes important. Diagnosis is made most accurately on culture of specimen however demonstration of actinomyces granules in histopathological sections from tissues not connected to hollow organs is strongly supportive of diagnosis ${ }^{[5]}$. In our case study we found on histopathological examination presence of inflammatory infiltrate in form of lymphocytes, plasma cells and foamy histiocytes. Characteristic sulfur granules were found in the tissue sections. Club shaped filamentous organism's were seen which was further confirmed on special stains. No neoplastic/atypical cells were seen. Hence our findings were supportive of the diagnosis of actinomycosis. Our histopathological findings were in concordance with paik et al. and Raymond et al.

The histopathological appearance of actinomycosis is similar in the various organs. These lesions have an outer zone of granulation tissue and a central abscess surrounding Actinomyces colonies. In lymph nodes, histologic features suggestive of Actinomyces infection are fibrous thickening of the capsule, nodulation with fibrous bands, multiple abscesses with central loculation, and scattered neutrophilladen macrophages. Characteristic sulfur granules may be seen in some sections. Differential diagnosis considered while evaluating such cases is both infectious etiologies in form of cat scratch disease, lymphogranuloma venereum, syphilis etc. while on other hand malignancies should also be kept in mind. In contrast to cat-scratch disease and lymph granuloma venereum, palisading histiocytes are not noted around the microabscess. The prominent fibrosis of the lymph node capsule may mimic the fibrosis seen in syphilitic lymphadenitis but plasmacytic infiltration of the capsule is not prominent in actinomyces lymphadenitis1. Another most important differential diagnosis to be considered is nocardia, as it also shows presence of neutrophilic abcess with characteristic granules and splendore hoeppli phenomenon ${ }^{[6]}$. However differentiating features between the two is nocardia is gram positive while actinomyces is a gram negative bacteria, nocardia stains with Zeihl Neelson stain (decolorizes with $1 \%$ sulfuric acid) while actinomyces do not. Both are positive with grocott silver methamine stain but nocardia stains negative with PAS and actinomyces stain positive with PAS ${ }^{[6]}$.

Actinomycosis is usually treated successfully with longterm penicillin $G$ therapy ${ }^{[8]}$. Effective antimicrobial treatment for actinomycosis requires the identification and susceptibility testing of any associated gram-negative infection.

Our patient is kept under regular follow up and has responded well to the treatment. Actinomycosis-associated lymphadenopathy is a rare condition that may clinically simulate malignancy. It should always be included in the differential diagnosis in patients with poor dental hygiene. Appropriate diagnosis significantly affects the patient management.

\section{References}

1. Naveed DM, Farooqui K, Muneeb I, Hamad W. Actinomycosis-A Compact Clinical Review. MAR Pulmonology 2021;2(1).

2. Arik D. Actinomyces Lymphadenitis: Case Report/Aktinomiçes Lenfadeniti: Olgu Sunumu. Turkish Journal of Pathology 2013;29(1):80-2.

3. Valour F, Sénéchal A, Dupieux C, Karsenty J, Lustig S, Breton $\mathrm{P}$ et al. Actinomycosis: etiology, clinical features, diagnosis, treatment, and management. Infection and drug resistance 2014;7:183.

4. Thukral R, Shrivastav K, Mathur V, Barodiya A, Shrivastav S. Actinomyces: a deceptive infection of oral cavity. Journal of the Korean Association of Oral and Maxillofacial Surgeons 2017;43(4):282.

5. Alborzi A, Pasyar N, Nasiri J. Actinomycosis as a neglected diagnosis of mediastinal mass. Japanese journal of infectious diseases 2006;59(1):52.

6. Kakuta R, Hidaka H, Yano H, Miyazaki H, Suzaki H, Nakamura $\mathrm{Y}$ et al.. Identification of Actinomyces meyeri actinomycosis in middle ear and mastoid by $16 \mathrm{~S}$ rRNA analysis. Journal of medical microbiology 2013;62(8):1245-8.

7. Alborzi A, Pasyar N, Nasiri J. Actinomycosis as a neglected diagnosis of mediastinal mass. Japanese journal of infectious diseases 2006;59(1):52.

8. Tasaduq Fazili MD, McNeil MM. Actinomyces species (Actinomycoses). 